\section{Critérios e indicadores de resultados para a farmácia hospitalar brasileira utilizando o método Delfos}

\author{
Establishment of criteria and outcome indicators \\ for hospital pharmacies in Brazil using Delphos
}

\author{
${ }^{1}$ Escola Nacional de Saúde \\ Pública Sergio Arouca, \\ Fundação Oswaldo Cruz, \\ Rio de Janeiro, Brasil. \\ 2 Faculdade de Farmácia, \\ Universidade Estácio de Sá, \\ Rio de Janeiro, Brasil. \\ Correspondência \\ R. Magarinos-Torres \\ Departamento de \\ Ciências Biológicas, \\ Escola Nacional de Saúde \\ Pública Sergio Arouca, \\ Fundação Oswaldo Cruz. \\ Av. Brasil 4036, salas 915/916, \\ Rio de Janeiro, $R J$ \\ 21040-361, Brasil. \\ rmtorres@infolink.com.br
}

\begin{abstract}
The hospital pharmacy is responsible for several services which influence inpatient outcomes. This paper describes the construction of criteria and outcome indicators for Brazilian hospital pharmacies using Delphos, a consensus technique in which physical presence of specialists is not necessary. 22 specialists from 4 Brazilian regions participated. The entire process was anchored on the logical model previously constructed for the Brazilian hospital pharmacy. The four phases were conducted through e-mail contact. The first round of contributions identified 103 possible outcomes. These were screened and classified according to adequacy, relevance and measurability, producing 22 criteria that originated 20 outcome indicators, grouped in 7 separate aspects of quality of the pharmacy service. Initial contributions helped clarify four different log frame components. All of the outcomes considered by specialists in this study have been already described in the literature as structure and process indicators. Based on the results, we believe that the construction of composite indicators for the Brazilian hospital pharmacy based on the aspects of quality that emerged from the analysis would better depict these indicators' contribution to the evaluation of inpatient care.
\end{abstract}

Hospital Pharmacy Service; Delphi Technique; Inpatients
Rachel Magarinos-Torres 1,2

Claudia Garcia Serpa Osório-de-Castro 1

Vera Lucia Edais Pepe 1

\section{Introdução}

A farmácia hospitalar ocupa importante posição dentro do contexto assistencial do Sistema Único de Saúde (SUS), pois é responsável por diversas atividades relacionadas ao medicamento; instrumento terapêutico com forte impacto na saúde e no custo hospitalar 1,2 .

Desde 1992 entidades brasileiras apontam a necessidade de instrumentos que possibilitem avaliar serviços de farmácia inseridos em hospitais brasileiros, embora existam publicações internacionais destinadas à avaliação de serviços farmacêuticos em unidades de saúde de uma maneira geral 3,4 .

Em 2001 foi elaborado, por meio de revisão bibliográfica e consenso de especialistas, um modelo lógico específico para farmácia hospitalar brasileira, onde todas as atividades do serviço direcionadas a pacientes hospitalizados foram agrupadas e enunciadas na forma de dez componentes ${ }^{5}$. Para cada componente foi elucidado o respectivo objetivo, produto e efeito esperado 5 . Esta construção possibilitou em 2002/2003 a primeira investigação de caráter nacional, que desenhou o panorama da farmácia hospitalar brasileira, intitulada Diagnóstico da Farmácia Hospitalar no Brasil 6,7.

Cabe destacar que esta pesquisa, sob a ótica da avaliação de serviços, ateve-se a explorar estrutura e processos desenvolvidos. Não foram levantados, naquele momento, resultados, ou 
seja, mudanças geradas, pelo serviço, no cuidado à saúde dirigida ao paciente hospitalizado 7,8 . Desde o início, esta questão esteve alocada para um segundo momento, onde seriam realizados estudos de caso ${ }^{5}$.

Partindo do pressuposto de que todos os componentes enunciados no modelo lógico da farmácia hospitalar, independente da forma como são realizados, produzem resultados, sejam positivos ou negativos e de que, muitos deles são passíveis de mensuração, este artigo descreve o processo de construção de critérios e indicadores de resultado para o serviço de farmácia hospitalar brasileiro pelo método Delfos, visando instrumentalizar futuras avaliações na área, entre elas, o segundo momento do Diagnóstico da Farmácia Hospitalar no Brasil.

A opção por utilizar Delfos resulta da complexidade e da diversidade das práticas desenvolvidas neste setor no Brasil, bem como da restrita produção científica sobre o tema 7,9.

\section{Metodologia}

Delfos, também denominado Delphi, é um método de consenso de especialistas. Espera-se que um método de consenso seja, ao mesmo tempo, de fácil aplicação, permita igualmente a participação de todos os envolvidos e estimule ampla discussão das questões-objetivo ${ }^{10}$. A principal característica do Delfos é a inexistência de contato presencial entre os participantes. A interação entre os membros é conseguida através da circulação das respostas individuais, sistematizadas sob a forma de resumo, enviado a cada nova interrogação. Um processo estruturado de retroalimentação mediante análise parcial dos dados coletados em cada rodada 11 .

Internacionalmente, os primeiros estudos conduzidos por este método na área da saúde focalizavam demandas educacionais, principalmente relacionadas a médicos e enfermeiros 12,13,14,15. Atualmente são encontradas também investigações com o propósito de obter consenso sobre condutas clínicas e adequação de indicadores 16,17 . No âmbito da assistência farmacêutica brasileira, Delfos já foi utilizado para elucidar questões políticas e padrões de qualidade para farmácias comerciais e drogarias 18,19

Neste estudo, o método Delfos esteve centrado na comunicação escrita por e-mail. Foram enviados quatro instrumentos denominados Documento de Trabalho. Embora, o objetivo inicial de cada Documento de Trabalho tenha sido definido a priori, sua formulação foi apontada ao longo do estudo. A Figura 1 apresenta esque- maticamente as etapas adotadas na coleta dos dados, bem como o número de comunicações devolvidas frente às enviadas.

\section{Instrumentos utilizados}

O Primeiro Documento de Trabalho teve como objetivo elucidar os resultados da farmácia hospitalar. O desejo, neste momento, era estimular um brainstorm centrado no foco da pesquisa. Dentre os pressupostos apresentados, admitiram-se como resultados da farmácia hospitalar, mudanças positivas ou negativas ocasionadas por atividades deste setor no cuidado à saúde de pacientes hospitalizados.

O texto enviado aos participantes solicitava resposta a quatro questões de trabalho, uma fechada e três abertas. As duas primeiras perguntas tinham como foco perceber se o modelo lógico da farmácia hospitalar contemplava todas as atividades necessárias para garantir a disponibilidade de medicamentos aos pacientes hospitalizados de forma racional. O modelo lógico apresentado aos participantes agregava revisão das definições dos objetivos de cada componente 9 . A terceira e a quarta questão solicitavam enunciar resultados para a farmácia hospitalar. Todas as sugestões recebidas foram observadas e, na medida do possível, considerando o escopo do estudo, ajustadas e agrupadas em resultados sugeridos.

O Segundo Documento de Trabalho apresentava resumo das considerações enviadas pelos participantes no Primeiro Documento de Trabalho e duas questões na forma de tabelas. A primeira relacionava resultados sugeridos para a categoria "conjunto de componentes da farmácia hospitalar" e a segunda, resultados direcionados a cada componente específico. O objetivo desta comunicação era localizar, dentre os resultados sugeridos, os adequados, e dentre estes, os relevantes e factíveis de serem mensurados. O julgamento dos itens quanto à adequação e ser passível de mensuração aceitava como resposta apenas "sim" ou "não". De forma distinta, a opinião quanto à relevância poderia ser ponderada através de valores, admitindo 0 (zero) para irrelevantes, 1 (um) para pouco relevante e 2 (dois) para os relevantes.

O terceiro momento, identificado como Segundo Documento de Trabalho Fase II retomou o Segundo Documento de Trabalho com o propósito de ampliar o consenso a respeito da adequação, da mensuração e da relevância dos resultados sugeridos anteriormente. Este instrumento forneceu resumo das contribuições obtidas no Segundo Documento de Trabalho, maiores escla- 


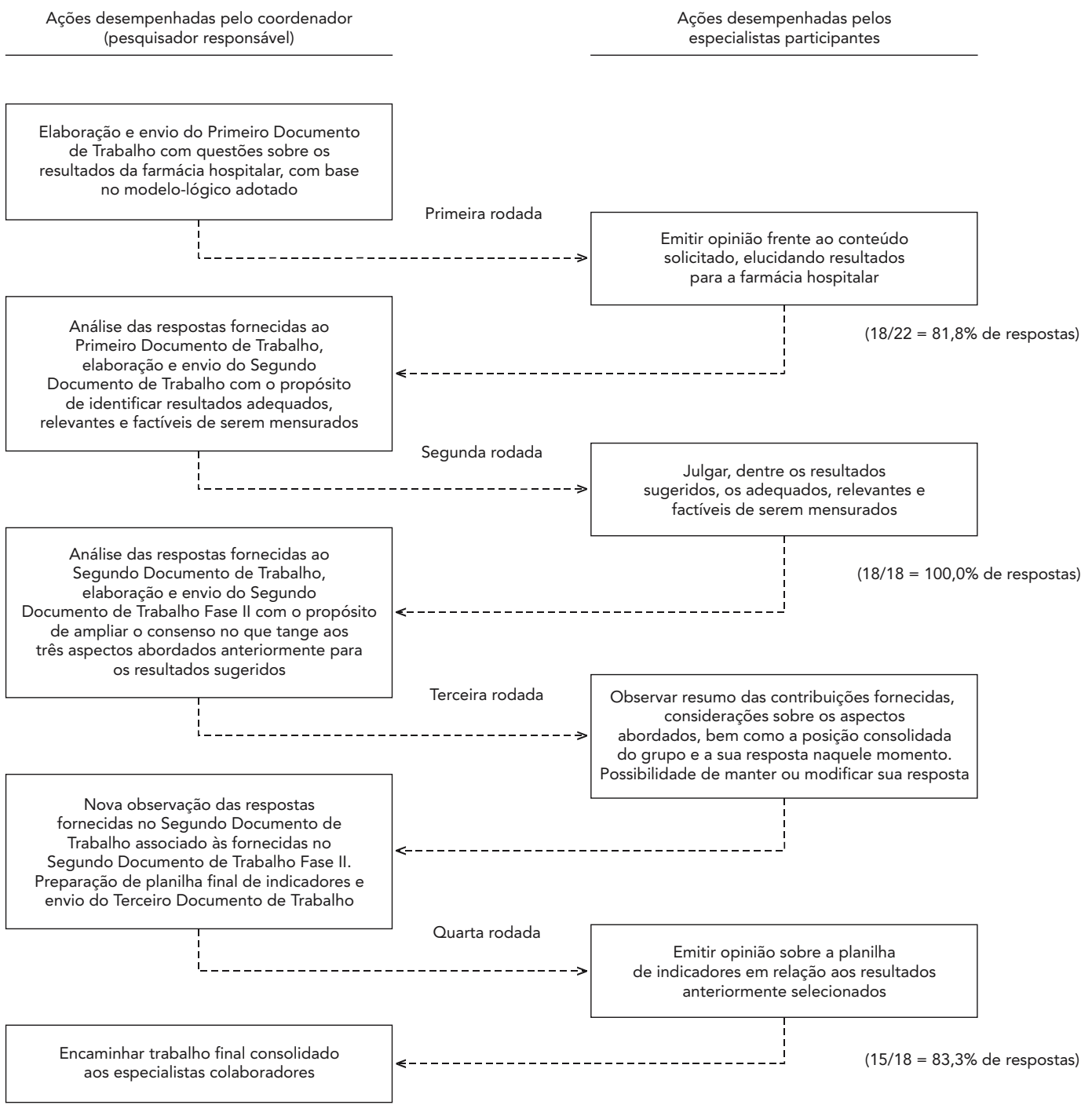

Adaptado de Rainhorn et al. 22.

recimentos sobre os requisitos considerados, a posição consolidada do grupo e a resposta apostada pelo especialista naquele momento.

Todas as respostas fornecidas no Segundo Documento de Trabalho e no Segundo Documento de Trabalho Fase II foram sistematizadas e analisadas com auxílio de algoritmo baseado em árvore de decisão. O objetivo foi observar simultaneamente, para os resultados inicialmente enunciados, independentemente do componente da farmácia hospitalar que os suscitou, os três requisitos abordados. O algoritmo foi construído atribuindo maior peso à adequação, seguido da relevância e por fim, de ser passível de mensuração. A aplicação do algoritmo resultou em uma grade classificatória de acordo com pontuação atingida por cada resultado. Essa grade foi hierarquizada pela pontuação e os primeiros $20 \%$ da distribuição constituíram-se dos resultados selecionados.

Por último, foi enviado aos especialistas o Terceiro Documento de Trabalho. O texto continha 
os resultados contemplados até o percentil 20 da distribuição total e a denominação proposta para indicadores correspondentes. Este instrumento forneceu também definição conceitual de todos os termos técnicos utilizados na denominação dos indicadores.

\section{Composição do grupo}

Participaram do estudo 22 profissionais de saúde de quatro das cinco regiões do Brasil com no mínimo dez anos de experiência em hospitais brasileiros e/ou com trabalhos publicados relacionados a atividades da farmácia hospitalar brasileira. Quanto à formação inicial, o grupo contou com farmacêuticos, médicos e enfermeiros. A participação foi firmada por Termo de Consentimento Livre e Esclarecido elaborado com base na Resolução $n^{o}$. 196/96 e aprovado pelo Comitê de Ética em Pesquisa do Instituto Fernandes Figueira.

\section{Resultados}

Para 78\% dos 18 especialistas que responderam o Primeiro Documento de Trabalho, todas as atividades da farmácia hospitalar encontram-se contempladas nos componentes enunciados pelo modelo lógico utilizado como referencial teórico, a saber: gerenciamento, seleção, programação, aquisição, armazenamento, distribuição, informação, seguimento farmacoterapêutico, farmacotécnica e ensino/pesquisa.

Os demais membros do painel sugeriram como novas atividades condizentes com o escopo do estudo a atenção farmacêutica, farmacovigilância, controle de estoque, participação em equipes multidisciplinares e sistema de garantia de qualidade. As contribuições permitiram explicitar melhor o objetivo do gerenciamento, armazenamento, seguimento farmacoterapêutico e ensino/pesquisa (Tabela 1). O estudo prosseguiu admitindo que todas as atividades do setor direcionadas a pacientes hospitalizados encontramse agrupadas nos componentes inicialmente enunciados.

Quanto aos resultados, foram sugeridos 63 itens para a categoria "conjunto de componentes da farmácia hospitalar” e, em média, 59,2 resultados específicos para cada componente. Três colaboradores assinalaram que os resultados para o conjunto de componentes deveriam ser a reunião dos citados para cada componente.

A análise das contribuições enviadas resultou em 103 itens. Destes, 14 relacionados ao conjunto de componentes, e em média, 8,6 para cada componente. O gerenciamento foi o componente com o maior número de resultados distintos sugeridos, 15. Em contrapartida, a farmacotécnica foi o que suscitou menor número, apenas quatro.

O julgamento dos especialistas, no que tange à adequação desses resultados ao serviço de farmácia hospitalar brasileiro, obteve concordância de $100 \%$ em 18 dos 103 itens. Certos resultados atingiram níveis de consenso de $94,4 \%$. Cabe destacar que $45 \%$ dos itens que obtiveram $100 \%$ de concordância quanto à adequação atingiram índices baixos nos aspectos "relevante de ser mensurado" e/ou "passível de mensuração". Foi possível identificar resultados considerados adequados por todos os membros do grupo, para 9 dos 10 componentes da farmácia hospitalar (Tabela 2).

A aplicação do algoritmo identificou 22 itens com pontuação dentro do percentil 20 (Tabela 3). Desses, quatro advindos do gerenciamento, seis da programação, três da aquisição, dois do armazenamento, dois do seguimento farmacoterapêutico e um cada para distribuição e ensino/ pesquisa. Seleção, informação e farmacotécnica não contribuíram individualmente. Três resultados citados para a categoria "conjunto de componentes da farmácia hospitalar” foram contemplados.

A percepção de que alguns destes 22 resultados estavam associados ao mesmo aspecto de qualidade do serviço possibilitou agregá-los e enunciá-los subdivididos em sete desses aspectos (Tabela 4). A seguir, foram denominados vinte indicadores correspondentes.

Cerca de $60 \%$ dos 15 especialistas que retornaram o Terceiro Documento de Trabalho sugeriram modificações. A maioria direcionada a aprimorar as definições dos termos presentes na denominação dos indicadores. A Tabela 4, presente neste artigo, já completa estas sugestões. Não houve dissenso quanto aos resultados selecionados com auxílio do algoritmo.

Apenas um participante sugeriu alteração no título de um aspecto da farmácia hospitalar a ser avaliado. Inicialmente descrito como: relacionados aos "problemas relacionados aos medicamentos". Segundo ele, a redação final mais adequada seria: relacionados a "resultados da farmacoterapia”. Sua justificativa apóia-se no receio quanto ao termo "problemas relacionados aos medicamentos" limitar a utilização dos indicadores correspondentes a hospitais que realizam atenção farmacêutica, uma vez que foi divulgado no Brasil através do Consenso Brasileiro de Atenção Farmacêutica 20.

No que concerne à denominação dos indicadores, foram recebidas considerações de 4 (33\%) membros do grupos. Os indicadores que suscitaram polêmica foram os relacionados a "proble- 
Componentes da farmácia hospitalar com respectivos objetivos: enunciados a priori e após considerações dos participantes do Delfos.

\begin{tabular}{|c|c|c|}
\hline Componentes & Objetivos enunciados anteriormente & Objetivos após Delfos \\
\hline Gerenciamento & $\begin{array}{l}\text { Prover estrutura organizacional e infra-estrutura } \\
\text { que viabilizem as ações do serviço de farmácia. }\end{array}$ & $\begin{array}{l}\text { Prover estrutura organizacional e infra-estrutura que viabilizem } \\
\text { as ações do serviço de farmácia, com a devida qualidade. }\end{array}$ \\
\hline Seleção & $\begin{array}{l}\text { Definir os medicamentos necessários para suprir as } \\
\text { necessidades do Hospital segundo critérios de eficácia } \\
\text { e segurança. Seguidos por qualidade, comodidade } \\
\text { posológica e custo. }\end{array}$ & $\begin{array}{l}\text { Definir os medicamentos necessários para suprir as } \\
\text { necessidades do hospital segundo critérios de eficácia } \\
\text { e segurança. Seguidos por qualidade, comodidade } \\
\text { posológica e custo. }\end{array}$ \\
\hline \multicolumn{3}{|l|}{ Logística } \\
\hline Programação & $\begin{array}{l}\text { Definir especificações técnicas e quantidades dos } \\
\text { medicamentos a serem adquiridos, tendo em vista os } \\
\text { recursos e prazos disponíveis. }\end{array}$ & $\begin{array}{l}\text { Definir especificações técnicas e quantidades dos } \\
\text { medicamentos a serem adquiridos, tendo em vista os } \\
\text { recursos e prazos disponíveis. }\end{array}$ \\
\hline Aquisição & $\begin{array}{l}\text { Suprir a demanda de medicamentos do hospital, tendo } \\
\text { em vista a qualidade. }\end{array}$ & $\begin{array}{l}\text { Suprir a demanda de medicamentos do hospital, } \\
\text { tendo em vista a qualidade. }\end{array}$ \\
\hline Armazenamento & $\begin{array}{l}\text { Manter disponibilidade de estoques, assegurando a } \\
\text { qualidade dos produtos. }\end{array}$ & $\begin{array}{l}\text { Manter disponibilidade de estoques, assegurando a } \\
\text { qualidade dos produtos. O que requer controle de estoque. }\end{array}$ \\
\hline Distribuição & $\begin{array}{l}\text { Fornecer medicamentos em condições adequadas e } \\
\text { tempestivas com garantia de qualidade do processo. }\end{array}$ & $\begin{array}{l}\text { Fornecer medicamentos em condições adequadas e } \\
\text { tempestivas com garantia de qualidade do processo. }\end{array}$ \\
\hline Informação & $\begin{array}{l}\text { Disponibilizar informação independente, objetiva e } \\
\text { apropriada sobre medicamentos e seu uso racional a: } \\
\text { pacientes, profissionais de saúde e gestores hospitalares. }\end{array}$ & $\begin{array}{l}\text { Disponibilizar informação independente, objetiva e apropriada } \\
\text { sobre medicamentos e seu uso racional a: pacientes, } \\
\text { profissionais de saúde e gestores hospitalares. }\end{array}$ \\
\hline $\begin{array}{l}\text { Seguimento } \\
\text { farmacoterapêutico }\end{array}$ & $\begin{array}{l}\text { Acompanhar o uso de medicamentos prescritos a cada } \\
\text { paciente individualmente. }\end{array}$ & $\begin{array}{l}\text { Acompanhar o uso de medicamentos prescritos a cada paciente } \\
\text { individualmente, com registro sistemático das atividades. }\end{array}$ \\
\hline Farmacotécnica & $\begin{array}{l}\text { Elaborar preparações magistrais e oficinais, disponíveis } \\
\text { ou não no mercado, e/ou fracionar especialidades } \\
\text { farmacêuticas para atender as necessidades dos } \\
\text { pacientes, resguardando a qualidade. }\end{array}$ & $\begin{array}{l}\text { Elaborar preparações magistrais e oficinais, disponíveis ou não } \\
\text { no mercado, e/ou fracionar especialidades farmacêuticas } \\
\text { para atender as necessidades dos pacientes, resguardando } \\
\text { a qualidade. }\end{array}$ \\
\hline Ensino/Pesquisa & $\begin{array}{l}\text { Formar recursos humanos para a farmácia hospitalar e } \\
\text { para a assistência farmacêutica. Produzir informação e } \\
\text { conhecimento que subsidiem o aprimoramento das } \\
\text { condutas e práticas vigentes. }\end{array}$ & $\begin{array}{l}\text { Formar recursos humanos para a farmácia hospitalar e para a } \\
\text { assistência farmacêutica. Produzir informação e conhecimento } \\
\text { que subsidiem o aprimoramento das condutas e práticas, } \\
\text { vigentes incluindo farmacovigilância e estudos de utilização } \\
\text { de medicamentos. }\end{array}$ \\
\hline
\end{tabular}

mas relacionados aos medicamentos". Para três especialistas a mensuração dos resultados do serviço no que tange a este aspecto deveria obter separadamente dados de erros de medicação, evento adverso, reação adversa a medicamentos, ocorrência de intervenção farmacêutica e problemas relacionados a medicamentos.

\section{Discussão}

Os resultados apresentados neste estudo serão discutidos sob duas perspectivas: confiabilida- de dos dados obtidos e critérios/indicadores apontados pelo consenso para avaliar o serviço de farmácia hospitalar brasileiro a partir de seus resultados.

\section{Confiabilidade do consenso}

O alto índice de concordância obtido em todas as etapas da aplicação do método confere validade ao consenso 21 .

Estudos que discutem Delfos como metodologia também destacam como importantes, além do grau de concordância, questões relacionadas 
Resultados enunciados que obtiveram $100 \%$ de concordância quanto à adequação, ordenados por componente da farmácia hospitalar.

Componentes da farmácia hospitalar

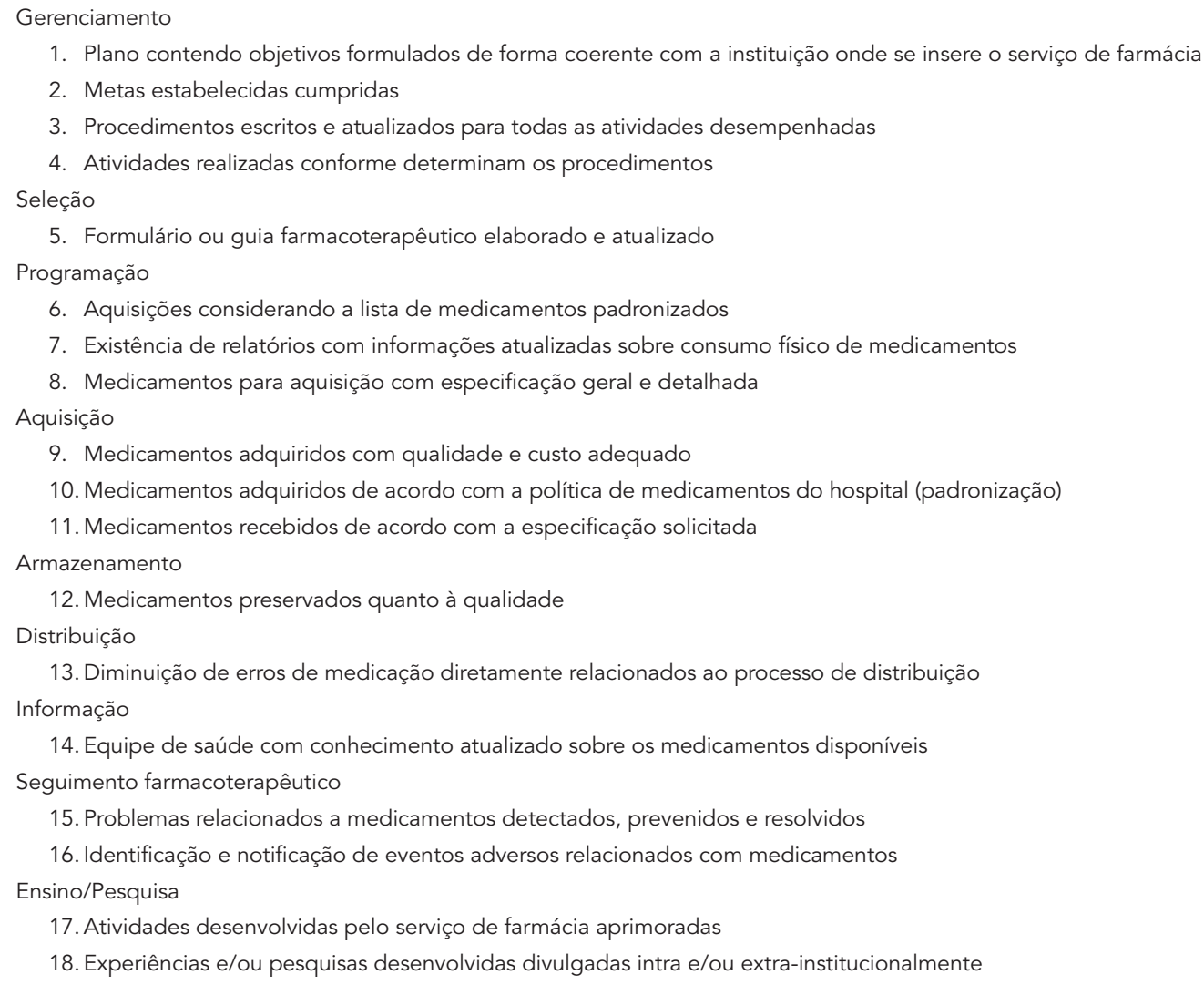

à condução do método, como: composição do grupo de especialistas, formulação dos instrumentos utilizados na coleta de dados, número de rodadas e perdas ao longo do estudo 9,10.

Embora não tenha sido possível, como inicialmente previsto, compor o grupo proporcionalmente de acordo com a distribuição de hospitais no Brasil, a formação final contou com representantes de quatro regiões brasileiras, sendo 16 da Região Sudeste e 2 de cada uma das regiões: Nordeste, Centro-Oeste e Sul. A presença de um número maior de especialistas do Sudeste condiz com o índice de publicação no âmbito da farmácia hospitalar advinda desta região. Além disso, a Região Sudeste é também a que concentra a maior rede hospitalar pública do país. Quanto à formação inicial o estudo contou com farmacêuticos, médicos e enfermeiros. Destes, cerca de $53 \%$ com mestrado e $27 \%$ doutorado.
Os instrumentos utilizados na condução do Delfos expressavam claramente seu objetivo. Com exceção do primeiro, que apresentava os pressupostos teóricos do estudo, os demais incluíram resumo das respostas fornecidas pelos participantes, referências bibliográficas correspondentes e considerações resultantes da análise parcial dos dados coletados. Todos os documentos enviados continham ao menos uma questão aberta onde o especialista poderia, caso percebesse necessidade, tecer considerações não contempladas nas questões enunciadas.

Uma vez que, neste estudo, a definição dos critérios configura-se como questão crucial para elaboração de indicadores, foram utilizadas duas rodadas no julgamento dos resultados enunciados para o serviço de farmácia hospitalar quanto à adequação, a ser passível de mensuração e à relevância da mensuração. 
Resultados com maior índice de concordância para os três critérios abordados (adequado, passível de mensuração e relevante de ser mensurado).

Resultados selecionados para construção de indicadores

1. Existência de lista de fornecedores qualificados avaliados tecnicamente

2. Medicamentos adquiridos de acordo com a política de medicamentos do hospital (padronização)

3. Medicamentos recebidos de acordo com a especificação solicitada

4. Medicamentos perdidos por caducidade ou falta de qualidade

5. Medicamentos recebidos em concordância com o parecer técnico

6. Ocorrência de reações adversas a medicamentos

7. Ocorrência de erros de medicação dispensação

8. Medicamentos perdidos (número de unidades e valores)

9. Diminuição de erros de medicação diretamente relacionados ao processo de distribuição

10. Recursos humanos atualizados e capacitados para o desenvolvimento das atividades de assistência farmacêutica em farmácia hospitalar

11. Participação e atuação dos farmacêuticos em comissões institucionais

12. Metas estabelecidas cumpridas

13. Procedimentos operacionais escritos e atualizados para todas as atividades desempenhadas

14. Atividades realizadas conforme determinam os procedimentos operacionais

15. Medicamentos disponíveis nas quantidades necessárias

16. Medicamentos para aquisição com especificação geral e detalhada

17. Medicamentos disponíveis em tempo oportuno

18. Aquisições considerando a lista de medicamentos padronizados

19. Existência de relatórios com informações atualizadas sobre consumo físico de medicamentos

20. Medicamentos dentro do prazo de validade

21. Problemas relacionados a medicamentos detectados, prevenidos e resolvidos

22. Identificação e notificação de eventos adversos relacionados com medicamentos

O número de participantes, bem como de rodadas e de perdas ao longo do trajeto, atingiu níveis considerados adequados quando comparados com outras investigações que utilizaram Delfos, incluindo experiências brasileiras. Grande parte dos estudos conseguiu obter consenso após envio de três comunicações. O tempo utilizado no processo variou de seis meses a um ano. São relatadas perdas mínimas de $6 \%$ e máxima de $43 \%$ dos participantes iniciais 15,16,17,18,19,21,22,23. Neste trabalho, foram realizadas quatro rodadas de questionários ao longo de dez meses. Responderam a todos os comunicados 15 especialistas do total de 22 que iniciaram no estudo, aproximadamente $68 \%$. Este estudo teve, portanto, uma perda de $32 \%$ dos especialistas.

\section{Critérios e indicadores apontados pelo consenso}

A principal contribuição deste estudo está relacionada à forma como os indicadores foram construídos, a partir da discussão dos critérios, observando o objetivo de cada componente da farmácia hospitalar 24. Desta maneira, torna-se mais clara a relação entre o que se pretende avaliar e o indicador a ser utilizado, bem como entre os dados obtidos a partir do cálculo destes indicadores e os pontos que necessitam de aprimoramento. Pelo mesmo motivo, foi oportuna a opção de re-agregar os indicadores segundo aspectos da qualidade do serviço a ser avaliado.

O maior número de resultados sugeridos para o componente gerenciamento na primeira rodada de questionários, frente ao menor para a farmacotécnica, parece estar diretamente relacionado ao diferente grau de compreensão sobre estes componentes. Parece que quanto mais claro o papel do componente no ambiente hospitalar, maior consenso sobre os resultados e, conseqüentemente, menor o número de resultados distintos citados. O maior entendimento sobre a farmacotécnica pode ser explicado pelo fato de que, historicamente, o papel do farmacêutico hospitalar aparece fortemente atrelado à produção de medicamentos 25 . Os dois primeiros livros brasileiros destinados especificamente à farmácia hospitalar incluem dentro do seu escopo a 
Denominação dos indicadores para a farmácia hospitalar, agrupados segundo aspecto a ser avaliado.

\begin{tabular}{|c|c|}
\hline Aspecto a ser avaliado & Denominação dos indicadores \\
\hline Relacionado à perda de medicamentos & $\begin{array}{l}\text { 1. Número de unidades de medicamentos perdidas } \\
\text { 2. Valor gasto com medicamentos perdidos }\end{array}$ \\
\hline $\begin{array}{l}\text { Relacionado à garantia da qualidade } \\
\text { dos medicamentos }\end{array}$ & $\begin{array}{l}\text { 3. Número de medicamentos especificados para a compra em } \\
\text { adequação à lista de medicamentos selecionados } \\
\text { 4. Número de medicamentos dentro do prazo de validade } \\
\text { 5. Existência de lista de fornecedores qualificados avaliados tecnicamente } \\
\text { 6. Número de medicamentos recebidos em adequação ao parecer técnico } \\
\text { 7. Número de medicamentos adquiridos em adequação à lista de } \\
\text { medicamentos selecionados }\end{array}$ \\
\hline $\begin{array}{l}\text { Relacionado à garantia da } \\
\text { disponibilidade dos medicamentos }\end{array}$ & $\begin{array}{l}\text { 8. Existência de relatórios com informações atualizadas sobre } \\
\text { consumo físico } \\
\text { 9. Número de medicamentos disponíveis em tempo oportuno } \\
\text { 10. Número de medicamentos disponíveis nas quantidades necessárias } \\
\text { 11. Número de medicamentos adquiridos em adequação à lista de } \\
\text { medicamentos selecionados }\end{array}$ \\
\hline & $\begin{array}{l}\text { 12. Número de medicamentos programados para a aquisição com } \\
\text { especificação detalhada } \\
\text { 13. Número de medicamentos adquiridos em adequação à especificação } \\
\text { solicitada }\end{array}$ \\
\hline Relacionados aos recursos humanos & $\begin{array}{l}\text { 14. Número de profissionais atuantes na farmácia hospitalar atualizados e } \\
\text { capacitados para o conjunto das atividades relacionadas à assistência } \\
\text { farmacêutica hospitalar } \\
\text { 15. Número de profissionais vinculados à farmácia hospitalar atuando em } \\
\text { comissões institucionais }\end{array}$ \\
\hline Relacionados às metas estabelecidas & 16. Número de metas estabelecidas cumpridas \\
\hline $\begin{array}{l}\text { Relacionados aos procedimentos } \\
\text { operacionais }\end{array}$ & $\begin{array}{l}\text { 17. Existência de procedimentos operacionais escritos e atualizados para } \\
\text { todas as atividades da farmácia hospitalar } \\
\text { 18. Número de itens dos procedimentos operacionais realizados } \\
\text { adequadamente }\end{array}$ \\
\hline $\begin{array}{l}\text { Relacionados a problemas } \\
\text { relacionados a medicamentos }\end{array}$ & $\begin{array}{l}\text { 19. Número de problemas ocorridos relacionados a medicamentos } \\
\text { 20. Número de problemas relacionados a medicamentos identificados e } \\
\text { notificados }\end{array}$ \\
\hline
\end{tabular}

manipulação/produção de medicamentos, mas, não o gerenciamento 26,27 .

Adespeito dos resultados que obtiveram $100 \%$ de concordância no que tange a "ser adequado", observa-se que todos encontram-se descritos na literatura seja como estrutura ou processo, atrelados ou não ao mesmo componente que este estudo 7,8,28. Isso reforça a idéia de interdependência entre os componentes, citado no início do Delfos por três dos colaboradores como justificativa para não responder à questão que solicitava resultados para a categoria "conjunto de componentes da farmácia hospitalar”, indican- do que os resultados para essa categoria seriam provenientes da reunião dos mencionados por componente. Postula-se que os resultados não se reportam apenas ao componente que o suscitou. Essa afirmação encontra corroboração em outro recente trabalho que apresenta fundamentos quanto à interdependência das atividades 29 .

Soma-se a isto a constatação de que julgar os resultados para o conjunto de componentes obteve maior índice de discordância entre os participantes. Aparentemente parece ser mais difícil pensar o serviço em sua forma mais complexa, até porque a realidade nos mostra que somen- 
te um número restrito de farmácias hospitalares realiza todas as atividades presentes no modelo lógico 7 .

Como o propósito desta investigação era identificar não somente resultados apropriados, como relevantes e passíveis de serem mensurados, em um contexto de prática habitual, sem a necessidade de modificações na conduta diária dos serviços de farmácia hospitalar, a análise simultânea das respostas fornecidas para estes requisitos pelo algoritmo possibilitou traduzir melhor a opinião do grupo quanto aos critérios mais importantes.

Chama atenção nenhum resultado dos enunciados para os componentes seleção, informação e farmacotécnica ter sido contemplado dentre os primeiros $20 \%$ da classificação final. Para os especialistas, estes componentes contribuem sim, mas de modo menos importante. Por outro lado, os resultados foram agregados sob aspectos da qualidade do serviço e não mais com relação direta a componentes específicos. Assim, não os componentes, mas seus resultados, estariam embutidos no panorama final.

Outro ponto de discussão diz respeito às conseqüências de alterar o título do aspecto a ser avaliado inicialmente descrito como relacionados a "problemas relacionados aos medicamentos" para relacionados a "resultados da farmacoterapia”. A princípio, esta modificação parece mais apropriada. Tampouco suscitaria alteração nos indicadores denominados, nem a inclusão de outros, uma vez que o termo "problemas relacionados aos medicamentos" cobre todos os possíveis resultados vinculados à farmacoterapia. A definição de "problemas relacionados aos medicamentos" abarca tanto erros de medicação como evento adverso, que por sua vez engloba reação adversa a medicamentos 30,31,32.

Nota-se que existe uma associação entre os diferentes aspectos de qualidade enunciados. Quanto menor a perda de medicamentos maior a disponibilidade de medicamentos. Da mesma forma, a garantia da qualidade dos medicamentos favorece a diminuição de "problemas relacionados aos medicamentos". Isto já estaria sinalizado na questão anteriormente citada, da interdependência de atividades 29 . Mas, parece lógico que ao abordar resultados do serviço, e não apenas estrutura e processo, a qualidade do serviço seja resultante de uma organicidade própria que seja favorecida pela boa qualidade das partes, ou resultados individuais 33 .

Avaliar essa qualidade de forma singular é difícil, principalmente no momento de traduzi-la em indicadores. Assim, cada aspecto da qualidade originou uma série de indicadores correspondentes. Buscou-se, para maior confiabilidade, corroboração na literatura. Todos os indicadores resultantes do processo de consenso deste trabalho já foram citados anteriormente em outras fontes 4,7,28,34,35. A maioria delas vincula o indicador a um componente e/ou a uma atividade.

Quando relacionados a aspectos da qualidade do serviço, como neste estudo, levanta-se a possibilidade de construção de indicadores compostos, um índice sintético capaz de mensurar de forma associada diferentes variáveis 36,37 . A construção de indicadores compostos para a farmácia hospitalar com base nos aspectos de qualidade permitiria delinear melhor os resultados do serviço.

Quanto à polêmica de mensurar separadamente erros de medicação, evento adverso e reação adversa cabe refletir sobre os ganhos, já que existe uma interseção entre estes desfechos. Avaliação de "problemas relacionados aos medicamentos", por sua vez, envolveria todos 30,31,32. Por outro lado, a ocorrência de intervenção farmacêutica no Brasil não se encontra exclusivamente atrelada à ocorrência de "problemas relacionados aos medicamentos". Pode se realizar uma intervenção farmacêutica para prevenir o aparecimento de problemas, mesmo quando o paciente não está exposto a fatores de risco 30 . Em virtude disto, todos os resultados selecionados na aplicação do algoritmo que continham estes termos foram agrupados.

\section{Considerações finais}

O consenso de 15 especialistas identificou 22 resultados da farmácia hospitalar brasileira que, agrupados em 7 aspectos da qualidade a serem avaliados, derivam em 20 indicadores.

A estratégia adotada, de abordar componente a componente para identificação dos resultados do serviço, foi oportuna não apenas para facilitar a elucidação pelo especialista colaborador, como também para discutir o modelo lógico da farmácia hospitalar. Permitiu ainda conhecer os critérios que o grupo de especialistas apontou como adequados para 9 dos 10 componentes da farmácia hospitalar.

Entretanto, estima-se que a avaliação dos resultados da farmácia hospitalar a partir dos aspectos de qualidade possibilite traduzir melhor sua contribuição no cuidado à saúde de pacientes hospitalizados do que a observação dos resultados por componente. Cada aspecto a ser avaliado requer análise do conjunto de indicadores correspondentes, o que aponta para a construção de indicadores compostos.

Os resultados e indicadores obtidos refletem a opinião do grupo de especialistas que parti- 
ciparam do consenso. No entanto, partindo-se da constatação de que o trabalho contou com especialistas brasileiros, foi construído sobre modelo lógico também produzido por consenso de especialistas no Brasil, aprovou indicadores já fartamente descritos na literatura e obteve alto índice de concordância em todas as suas etapas constitutivas, tenha cumprido seus objetivos e possua validade na avaliação de serviços de farmácia hospitalar no Brasil.

\section{Resumo}

A farmácia hospitalar é responsável por diversas atividades com influência no cuidado à saúde de pacientes hospitalizados. Este artigo descreve a construção de critérios e indicadores de resultado para a farmácia hospitalar brasileira utilizando Delfos, um método de consenso que dispensa contato presencial. Participaram do estudo 22 especialistas de quatro regiões brasileiras. Todo processo ancorou-se no modelo lógico da farmácia hospitalar brasileira, previamente construído. Foram enviados por correio eletrônico quatro documentos de trabalho. A primeira rodada identificou 103 possiveis resultados. O julgamento quanto à adequação, relevância e possibilidade de mensuração permitiu selecionar 22 critérios que originaram 20 indicadores, agrupados em 7 aspectos da qualidade do serviço. As contribuições iniciais permitiram explicitar melhor o objetivo de quatro componentes do modelo lógico. Observa-se que todos os itens considerados como resultado pelos especialistas neste estudo encontram-se descritos na literatura, seja como indicadores de estrutura ou processo da farmácia hospitalar. Estima-se que a construção de indicadores compostos para a farmácia hospitalar, com base nos aspectos de qualidade observados, permitiria traduzir melhor sua contribuição na avaliação do cuidado a pacientes hospitalizados.

Serviço de Farmácia Hospitalar; Técnica Delfos; Pacientes Internados

\section{Colaboradores}

R. Magarinos-Torres foi responsável pela revisão de literatura, elaboração da metodologia, coleta dos dados, análise dos resultados e redação do artigo final. C. G. S. Osório-de-Castro eV. L. E. Pepe orientaram a metodologia, aportaram conhecimentos e revisaram o artigo.

\section{Agradecimentos}

A todos os especialistas que participaram do grupo Delfos: Adriano Max Moreira Reis, Ângela Maria de Souza Ponciano, Edmara Schadeck, Francisco de Assis Acurcio, Ilenir Leão Tuma, Joice Mara Cruciol e Souza, Leticia Lemme, Lisiane da Silveira Ev, Márcia Azevedo Bastian Manfredi, Maria Lucia Rodrigues, Mario Borges Rosa, Neudo Magnago Heleodoro, Ranieri Carvalho Camuzi, Silvia Helena de Bortoli Cassiani e Sonia Lucena Cipriano (nomes autorizados em ordem alfabética). A Dra. Selma Rodrigues de Castilho pelas considerações ao longo do estudo. 


\section{Referências}

1. Departamento de Atenção Básica, Secretaria de Políticas de Saúde, Ministério da Saúde. Política nacional de medicamentos. Brasília: Ministério da Saúde; 2001. (Série C. Projetos, Programas e Relatórios, 25).

2. Organización Mundial de la Salud. Promoción del uso racional de medicamentos: componentes centrales. Perspectivas políticas sobre medicamentos de la OMS. Genebra: Organización Mundial de la Salud; 2002.

3. Organização Pan-Americana da Saúde/Ministério da Saúde/Conselho Federal de Farmácia, organizadores. Organização da assistência farmacêutica em hospitais - relatório final da oficina de trabalho. Brasília: Organização Pan-Americana da Saúde; 1992.

4. Organización Panamericana de la Salud. Programa regional de medicamentos esenciales: guía para el diagnóstico de farmacias y servicios farmacéuticos en las unidades de salud. Washington, DC; Organización Panamericana de la Salud; 1991.

5. Marin N, Cosendey MAE, Luiza VL, Osório-de-Castro CGS, Castro M, Castilho SR, et al. Projeto Diagnóstico da Farmácia Hospitalar no Brasil. Brasília: Organização Pan-Americana da Saúde/Núcleo de Assistência Farmacêutica, Escola Nacional de Saúde Pública Sergio Arouca, Fundação Oswaldo Cruz/Sociedade Brasileira de Farmácia Hospitalar/Conselho Federal de Farmácia; 2001.

6. Marin N, Osório-de-Castro CGS, Cosendey MAE, Luiza VL, Castro M, Castilho SR, et al. Projeto Diagnóstico da Farmácia Hospitalar no Brasil: relatório técnico final. Rio de Janeiro: Organização Pan-Americana da Saúde/Núcleo de Assistência Farmacêutica, Escola Nacional de Saúde Pública Sergio Arouca, Fundação Oswaldo Cruz/Sociedade Brasileira de Farmácia Hospitalar/Conselho Federal de Farmácia; 2003.

7. Osório-de-Castro C, Castilho SR, organizadores. Diagnóstico da Farmácia Hospitalar no Brasil. Rio de Janeiro: Editora Fiocruz; 2004.

8. Donabedian A. The role of autcomes in quality assessment and assurance. QRB Qual Rev Bull 1992; 18:356-60.

9. Spinola AWP. Técnica prospectiva Delphi: abordagem teórico-prática. São Paulo: SM Editora; 1997.

10. Jeremy J, Duncan H. Usando o Delfos e a técnica do grupo nominal na pesquisa em saúde. In: Pope C, Mays N, organizadores. Pesquisa qualitativa na atenção à saúde. 2a Ed. Porto Alegre: Editora Artmed; 2005. p. 51-60.

11. Keyes JA, Wilson MP, Becker J. The future of medical education: forecast of the Council of Deans. J Med Educ. 1975; 50:319-27.

12. Lindeman CA. Delphi survey of priorities in clinical nursing research. Nurs Res 1975; 24:434-41.

13. Abdallah MM, Evans AT. Educational planning for a urologic physician's assistant program by the Delphi method. J Allied Health 1975; 4:23-6.

14. Bramwell L, Hykawy E. The Delphi technique: a possible tool for predicting future events in nursing education. Nurs Pap 1974; 6:23-32.
15. Almasio PL, Niero M, Angioli D, Ascione A, Gullini S, Minoli G, et al. Experts' opinions on the role of liver biopsy in HCV infection: a Delphi survey by the Italian Association of Hospital Gastroenterologists (A.I.G.O.). J Hepatol 2005; 43:381-7.

16. Nieuwenhuijsen K, Verbeek JH, de Boer AG, Blonk RW, van Dijk FJ. Validation of performance indicators for rehabilitation of workers with mental health problems. Med Care 2005; 43:1034-42.

17. Marshall M, Lockwood A, Lewis S, Fiander M. Essential elements of an early intervention service for psychosis: the opinions of expert clinicians. BMC Psychiatry 2004; 4:17.

18. Chaves GC. O processo de implementação do acordo TRIPS da OMC em países da América Latina e Caribe: análise das legislações de propriedade industrial sob a ótica da saúde pública [Dissertação de Mestrado]. Rio de Janeiro: Escola Nacional de Saúde Pública, Fundação Oswaldo Cruz; 2005.

19. Silva RR. Acreditação de farmácias: a construção de um modelo [Tese de Doutorado]. São Paulo: Faculdade de Saúde Pública, Universidade de São Paulo; 2003.

20. Ivama AM, Noblat L, Castro MS, Oliveira NVBV, Jaramilo NM, Rech N. Consenso brasileiro de atenção farmacêutica: proposta. Brasília: Organização Pan-Americana da Saúde; 2002.

21. Rainhorn JD, Brudon-Jacobowicz P, Reich MR. Priorities for pharmaceutical polices in developing countries: results of a Delphi survey. Bull World Health Organ 1994; 72:257-84.

22. Telles Filho PCP, Cassiani SHB. Administração de medicamentos: aquisição de conhecimentos e habilidades requeridas por um grupo de enfermeiros. Rev Latinoam Enfermagem 2004; 12:533-40.

23. del Rocío Ortiz M, Álvarez-Dardet C, Ruiz MT, Gascón E. Identificación de barreras a las políticas de nutrición y alimentación en Colombia: estudio por el método Delfos. Rev Panam Salud Pública 2003; 14:186-92.

24. Akerman M, Nadanovsky P. Avaliação dos serviços de saúde: avaliar o quê? Cad Saúde Pública 1992; 8:361-5.

25. Gomes MJVM, Reis AMM. Farmácia hospitalar: histórico, objetivos e funções. In: Gomes MJVM, organizador. Ciências farmacêuticas: uma abordagem em farmácia hospitalar. São Paulo: Editora Atheneu; 2001. p 275-87.

26. Maia-Neto JF. Farmácia hospitalar: breve histórico. In: Maia-Neto JF, organizador. Farmácia hospitalar: um enfoque sistêmico. Brasília: Thesaurus Editora; 1995. p. 21-32.

27. Ministério da Saúde. Funções da farmácia hospitalar. In: Ministério da Saúde, organizador. Guia básico para a farmácia hospitalar. Brasília: Ministério da Saúde; 1994. p. 31-62.

28. Marin N, Luiza VL, Osório-de-Castro CGS, Machado dos-Santos S, organizadores. Assistência farmacêutica para gerentes municipais. Rio de Janeiro: Organização Pan-Americana da Saúde/Organização Mundial da Saúde; 2003. 
29. Messeder AM. Avaliação de estrutura e processo de serviços de farmácia hospitalar segundo nível de complexidade do hospital [Dissertação de Mestrado]. Rio de Janeiro: Escola Nacional de Saúde Publica, Fundação Oswaldo Cruz; 2005.

30. Organização Pan-Americana da Saúde Atenção farmacêutica no Brasil: trilhando caminhos: relatório 2001-2002. Brasília: Organização Pan-Americana da Saúde; 2002.

31. Rosa MB, Perini E. Erros de medicação: quem foi? AMB Rev Assoc Med Bras 2003; 49:335-41.

32. Agência Nacional de Vigilância Sanitária/Organização Pan-Americana da Saúde/Organização Mundial da Saúde. A importância da farmacovigilância: monitorização da segurança dos medicamentos. Brasília: Organização Pan-Americana da Saúde/Organização Mundial da Saúde; 2005.

33. Cosendey MAE, Luiza VL. Avaliação em saúde e políticas farmacêuticas. In: Bermudez JAZ, Oliveira MA, Escher A, organizadores. Acesso a medicamentos: direito fundamental, papel do Estado. Rio de Janeiro: Escola Nacional de Saúde Pública, Fundação Oswaldo Cruz; 2004. p. 157-74.
34. Cipriano SL. Proposta de um conjunto de indicadores para utilização na farmácia hospitalar com foco na acreditação hospitalar [Dissertação de Mestrado]. São Paulo: Faculdade de Saúde Pública, Universidade de São Paulo; 2004.

35. Organización Panamericana de la Salud. Guía para el desarrollo de servicios farmacéuticos hospitalarios. Washington DC: Organización Panamericana de la Salud; 1997. (Serie Medicamentos Esenciales y Tecnología).

36. Akerman M. Examinando elementos que possam influenciar a formulação de políticas em estudos que utilizaram indicadores compostos: o chão contra o cifrão. Ciênc Saúde Coletiva 2000; 5:11523.

37. Chiesa AN, Westphal MF, Kashiwagi NM. Geoprocessamento e a promoção da saúde: desigualdades sociais e ambientais em São Paulo. Rev Saúde Pública 2002; 36:559-67.

Recebido em 12/Abr/2006

Aprovado em 01/Mar/2007 